\title{
Comparison of Susceptibility of Clinical Isolates of Gram- Negative Bacilli to Cephalosporins and Fluroquinolone
}

\author{
Vishwajith*, K. Anuradha and D. Venkatesha \\ Department of Microbiology, Mysore Medical College and Research Institute, \\ Mysore- 570 001, India \\ *Corresponding author
}

Keywords

Cephalosporins, Ciprofloxacin, Gram negative bacilli, Antibiotic susceptibility test

Article Info

\section{Accepted:}

20 January 2021

Available Online:

10 February 2021
Cephalosporins and ciprofloxacin are commonly used in the treatment of gramnegative bacilli infections. Third generation cephalosporins are first choice but expensive than ciprofloxacin. So there is a need to compare their susceptibility. To compare the susceptibility of gram-negative bacilli clinical isolates to cephalosporins and ciprofloxacin. Susceptibility of ESBL and AmpC producers to ciprofloxacin. Disk diffusion method performed on100 Gram negative bacilli isolates. Susceptibility to cefuroxime, cefotaxime, ceftazidime, ceftazidime with clavulanic acid, cefoxitin, cefepime and ciprofloxacin were tested. ESBL detected by $>7 \mathrm{~mm}$ zone with ceftazidime clavulanic acid than ceftazidime alone. AmpC detected by cefoxitin resistant and cefepime sensitive. Among 100 isolates, 8 isolates were ESBL producers and 5 isolates were AmpC producers out of which $4(50 \%)$ isolates of ESBL and 2 isolates of AmpC producers were ciprofloxacin susceptible. Ciprofloxacin showed a susceptibility of 54\% in Pseudomonas, $72 \%$ in E. coli, $29 \%$ in Klebsiella, 66\% in Enterobacter, $80 \%$ in Citrobacter and complete resistance in Acinetobacter. Ciprofloxacin was found to be more active than the fourth generation cephalosporin cefepime against gram negative bacilli. Since ciprofloxacin are cheaper it reduces the treatment cost.

\section{Introduction}

Gram-negative bacteria are common causes of intra-abdominal infections (IAIs), urinary tract infections (UTIs), nosocomial pneumonia, and bacteremia.[1] The most common organisms responsible for these infections are multidrug resistant gram negative bacilli particularly members of the family Enterobacteriaceae and non fermenting gram negative rods [2]. Antimicrobial resistance among GNB is increasing worldwide. This is a major public health problem and a cause for both substantial morbidity and mortality among hospitalized patients. A direct correlation has been shown between resistance of GNB and patient mortality, cost of patient care and length of stay in the hospital [3]. 
Cephalosporins and ciprofloxacin are commonly used in the treatment of these infections. Cephalosporins are similar to penicillins, but more stable to many bacterial $\beta$ lactamases and therefore have a broader spectrum of activity. However, strains of $E$ coli and Klebsiella sp expressing extendedspectrum $\beta$ lactamases that can hydrolyze most cephalosporins are a growing clinical concern. Beside this Cephalosporins can cause hypersensitivity reactions that are identical to those of penicillins [4].

Fluoroquinolones were originally developed because of their excellent activity against gram-negative aerobic bacteria they had limited activity against gram-positive organisms. Further ciprofloxacin are cheap, and its half-life is 3- 5 hours with a bioavailability of $70 \%$ it can be orally administered twice daily. It is widely distributed in body fluids and tissues and well tolerated compared to cephalosporins[5].

In the Ambler structural classification of $\beta$ lactamases, AmpC enzymes belong to Class $\mathrm{C}$, while in the functional classification scheme of Bush these are assigned to group 3[6][7]. These enzymes can be chromosomal or plasmid encoded. AmpC $\beta$-lactamases are clinically significant,[8] since these confer resistance to cephalosporins in the oxyimino group (cefotaxime, ceftazidime, ceftriaxone), 7- $\alpha$ methoxy cephalosporins (cefoxitin or cefotetan) and are not affected by available $\beta$ lactamase inhibitors (clavulanate, sulbactam, tazobactam)[9]. Plasmid mediated AmpC $\beta$ lactamases differ from chromosomal AmpCs in being uninducible and are typically associated with broad multidrug resistance [10]. Plasmid mediated AmpC $\beta$-lactamases are present in isolates of Klebsiella pneumoniae, K. oxytoca, Salmonella spp., Proteus mirabilis, Escherichia coli, Citrobacter freundii and Enterobacter aerogenes [11]. In E. coli, high level production of chromosomally mediated AmpC $\beta$-lactamases is also present [12]. The treatment options for infections caused by organisms expressing AmpC $\beta$-lactamases are limited.

Resistance to extended-spectrum cephalosporins in E. coli and, in particular, $K$. pneumoniae has become a worldwide problem [13]. Dissemination of ESBL-producing Enterobacteriaceae in the community poses a new threat, since this may become a powerful reservoir for the continued influx of resistant strains into hospitals[14][15].

Thus there is a need for detecting AmpC $\beta$ lactamases so as to avoid therapeutic failures. The reports of AmpC $\beta$-lactamases from India are still limited. Therefore, there is a need to study for both extended spectrum $\beta$-lactamase (ESBL) and AmpC $\beta$-lactamases.

Resistance to Fluoroquinolones and cephalosorinsare increasing and there is a need to evaluate their resistance pattern. Keeping this in mind we decided to compare the susceptibility of gram negative bacilli clinical isolates to ciprofloxacin with that of second, third and fourth generation of cephalosorins.

\section{Objectives}

To compare the susceptibility of gram negative bacilli clinical isolates to cephalosporins and ciprofloxacin. Susceptibility of ESBL and AmpC producers to ciprofloxacin

\section{Materials and Methods}

Gram negative bacterial isolates from samples of exudate, sputum, blood, urine and burn patients were identified using the conventional methods and tested for Antibiotic susceptibility testing by Kirby Bauer method using cefuroxime, cefotaxime, ceftazidime, 
ceftazidime with clavulanic acid, cefoxitin, cefepime and ciprofloxacin as per CLSI guidelines[16].

ESBL producers were detected by disc diffusion method using ceftazidime and ceftazidime/clavulanic acid disc as per CLSI guidelines[16].

Ceftazidime-clavulanic acid disc was placed toward the center of the plate, a ceftazidime disc $(30 \mathrm{mg})$ was placed $15 \mathrm{~mm}$ out from the edge of ceftazidime-clavulanic acid disc at $90^{\circ}$ angles, so that it's inner edge was $15 \mathrm{~mm}$ from it. Plates were incubated at $35^{\circ} \mathrm{C}$, aerobically for 18-24 h. Organism was detected as ESBL by $>7 \mathrm{~mm}$ zone with ceftazidime clavulanic acid than ceftazidime alone.

AmpC producers were detected by disc diffusion method using cefoxitin and cefepime discs. Cefoxitin zone of $<18 \mathrm{~mm}$ was taken as cefoxitin resistant. Isolates resistant to cefoxitin and sensitive to cefepime was taken as $\mathrm{AmpC}$ producers.

\section{Results and Discussion}

A total of 100 Gram negative bacilli isolated from various clinical specimens were included. Among them 31 isolates were from exudates samples, 24 from burns, 19 from sputum, 16 from blood and 10 from urine samples (Table 1).

The organisms isolated are Klebsiella spp. 31(31\%), Escherichia coli 25 (25\%), Pseudomonas spp 24 (24\%), Enterobacter spp 12 (12\%), Citrobacter spp 5 (5\%), and Acinetobacter spp 3(3\%) (Table 2).

Pseudomonas was isolated frequently from burn wounds, Enterobacter from blood while Klebsiella spp from exudate and respiratory tract, Escherichia coli from urine specimens (Table 3). The susceptibility pattern of various isolates are shown in Table 4. All isolates showed good susceptibility to ciprofloxacin except Acinetobacter spp only one strain of it was sensitive to cefepime.

Among 100 isolates, 8 isolates were ESBL producers and 5 isolates were $\mathrm{AmpC}$ producers out of which $4(50 \%)$ isolates of ESBL and 2 isolates of AmpC producers were ciprofloxacin susceptible.

Klebsiella species isolates from exudates, burns and sputum showed susceptibility to ciprofloxacin 2(22\%), 1(11\%), 5(50\%) and cefepime $2(22 \%), \quad 1(50 \%), \quad 5(50 \%)$ respectively.

Escherichia coli susceptibility to ciprofloxacin and cefepime, blood isolates showed 100\% susceptibility whereas isolates from exudate showed 2(28\%), 4(57\%), from urine 6(66\%), $7(78 \%)$ respectively. Equal susceptibility to both $4(57 \%)$ was observed in sputum isolates.

Pseudomonas species isolated from various specimens showed susceptibility to ciprofloxacin and cefepime from exudate $4(57 \%), \quad 3(43 \%)$, burns 5(38\%), 4(31\%) respectively. Blood isolates were susceptible to cefepime $3(75 \%)$ other cephalosporins and ciprofloxacin $2(50 \%)$.

Enterobacter species isolated from exudate and burns showed $100 \%$ susceptibility tociprofloxacin, $4(67 \%)$ of blood isolates were susceptible. Only 4(67\%) blood isolates were cefepime susceptible.

Citrobacter species isolated from blood, urine were $100 \%$ sensitive to ciprofloxacin. Exudate isolate $1(33 \%)$ was sensitive to all cephalosporins and ciprofloxacin. Acinetobacter species isolated from exudate alone. Out of which only 1(33\%) was sensitive only to cefepime. All other antibiotics were resistant. 
Ciprofloxacin susceptibility seen in Pseudomonas 54\%, E.coli 72\%, Klebsiella 29\%, Enterobacter 66\%, Citrobacter $80 \%$ and Acinetobacter resistant.

Bacterial resistance to ceftazidime is a serious problem. Approximately 40\% of Enterobacter cloacae were resistant to ceftazidime, according to studies of ICU isolates in the United States between 1987 and 1991[17] and between 1994 and 1995[18]. In our study all Entrobacter spp were resistant to ceftazidime (Table 4).

This is probably related to production of stably derepressed chromosomal class- $1 \quad \beta-$ lactamase, which hydrolyzes $\beta$-lactam antibiotics other than carbapenems. Previous use of third-generation cephalosporins is more likely to cause the selection of resistance to $\beta$ lactams in blood isolates of Enterobacter species, which is associated with higher mortality[19].

An increased ciprofloxacin resistance among Enterobacter species was seen in Belgium and France compared with previous studies performed in 1990 [20] and 1991[21]. In our study $66 \%$ of Enterobacter spp. was sensitive to ciprofloxacin.

In Portugal and France, $36 \%$ and 26\%, respectively, of Klebsiella pneumoniae showed decreased susceptibility to ceftazidime. This was probably caused by the production of extended-spectrum $\beta$-lactamases [22] [23] and is concordant with a study by Livermore and Yuan [24]. According to Jarlier et al., [21] decreased susceptibility to ceftazidime among $K$. pneumoniae in French ICUs was 36\% in 1991. In the United States, resistance to ceftazidime among $K$. pneumoniae in ICU isolates increased from $3.6 \%$ in 1990 to $14.4 \%$ in 1993[25]. In our study Klebsiella spp showed 6\% sensitivity to ceftazidime.

The potency of cefepime, a parenteral aminothiazoly lmethoxyimino cephalosporin, was assessed against 256 ceftazidime-resistant Gram-negative bacilli from five medical centers in the United States. In addition, cefepime activity was compared with that of ciprofloxacin and imipenem against 506 ceftazidime-resistant Gram-negative bacilli collected during an 11-medical-center international study.

All US clinical isolates were susceptible ( $<$ or $=8 \mathrm{micrograms} / \mathrm{ml}$ ) to cefepime except Enterobacter cloacae (94\% susceptible) and Pseudomonas aeruginosa (19\% susceptible).

Enterobacteriaceae isolates from the 11-nation sample were $>80 \%$ cefepime susceptible with the exception of those from Brazil (48\% susceptible) and Italy (55\% susceptible [26].

These international, enteric isolates were also very susceptible to ciprofloxacin $(55 \%-100 \%$ susceptible). Nonenteric organisms (Pseudomonas, Xanthomonas and Acinetobacter) from the same international locations had overall rates of susceptibility of $47 \%$ for ciprofloxacin, and only 5\% for cefepime [26]. In our study Gram negative bacilli susceptibility ranged from $25-60 \%$ to cefepime and $29-80 \%$ for ciprofloxacin. Thus ciprofloxacin was more effective compared to cefepime.

A worrisome trend during the last two decades has been the development of resistance to extended-spectrum cephalosporins, e.g., cefotaxime, ceftazidime, and ceftriaxone. 
Table.1 Distribution of various clinical specimens

\begin{tabular}{|c|c|}
\hline EXUDATE & $\mathbf{3 1}$ \\
\hline BURNS & 24 \\
\hline SPUTUM & 19 \\
\hline BLOOD & 16 \\
\hline URINE & 10 \\
\hline TOTAL SAMPLES & 100 \\
\hline
\end{tabular}

Table.2 Isolates from various clinical specimens

\begin{tabular}{|c|c|}
\hline KLEBSIELLA SPP & $\mathbf{3 1}$ \\
\hline ESCHERICHIA COLI & 25 \\
\hline PSEUDOMONAS SPP & 24 \\
\hline ENTEROBACTER SPP & 12 \\
\hline CITROBACTER SPP & 5 \\
\hline ACINETOBACTER SPP & 3 \\
\hline TOTAL SAMPLES & 100 \\
\hline
\end{tabular}

Table.3 Distribution of various isolates in different clinical specimens

\begin{tabular}{|c|c|c|c|c|c|}
\hline & $\begin{array}{c}\text { Exud } \\
\text { ates }\end{array}$ & $\begin{array}{c}\text { Bur } \\
\text { ns }\end{array}$ & $\begin{array}{c}\text { Sput } \\
\text { um }\end{array}$ & $\begin{array}{c}\text { Blo } \\
\text { od }\end{array}$ & $\begin{array}{c}\text { Uri } \\
\text { ne }\end{array}$ \\
\hline PSEUDOMONAS & 7 & 13 & - & 4 & - \\
\hline E COLI & 7 & - & 7 & 3 & 9 \\
\hline KLEBSIELLA & 10 & 9 & 10 & 2 & - \\
\hline ENTEROBACTER & 2 & 2 & 2 & 6 & - \\
\hline CITROBACTER & 3 & - & - & 1 & 1 \\
\hline $\begin{array}{c}\text { ACINETOBACTE } \\
\text { R }\end{array}$ & 3 & - & - & - & - \\
\hline
\end{tabular}

Table.4 Susceptibility pattern of Gram negative bacterial isolates

\begin{tabular}{|c|c|c|c|c|c|}
\hline Organism & Cefotaxime & Cefuroxime & Ceftazidime & Cefepime & Ciprofloxacin \\
\hline Pseudomonas & $2(8.3)$ & $2(8.3)$ & $5(21)$ & $13(54)$ & $15(62.5)$ \\
\hline E Coli & $3(12)$ & $3(12)$ & $3(12)$ & $15(60)$ & $18(72)$ \\
\hline Klebsiella & $2(6)$ & $2(6)$ & $2(6)$ & $8(25)$ & $9(29)$ \\
\hline Enterobacter & - & - & - & $5(41.6)$ & $8(66)$ \\
\hline Citrobacter & $1(20)$ & $2(40)$ & $2(40)$ & $2(40)$ & $4(80)$ \\
\hline Acinetobacter & - & - & - & $1(33)$ & - \\
\hline
\end{tabular}


Such resistance is most often due to the breakdown of the extended-spectrum cephalosporin by extended-spectrum $\beta$ lactamases (ESBLs), but it may also be due to plasmid-mediated or chromosomally hyperproduced AmpC[27].

Failure to detect $\mathrm{ES} \beta \mathrm{L}-$ and AmpC $\beta \mathrm{L}-$ producing strains has contributed to their uncontrolled spread and therapeutic failures. Hence, their appearance in a hospital setting should be identified quickly so that appropriate antibiotic usage and containment measures can be implemented. For detection of various types of ES $\beta$ Ls, molecular methods are the best but the facilities are not available especially in the developing countries. Therefore, various phenotypic methods are recommended for routine use to detect $\mathrm{ES} \beta \mathrm{L}$ production in Gram-negative bacilli[28]. ESBL producers were $8(8 \%)$ and AmpC $\beta \mathrm{L}$ producers were $5(5 \%)$ in our study. Resistance to fluoroquinolones is frequently observed among ESBL producers [27]. In our study among ESBL producers 4(50\%) and among AmpC producers 2(40\%) were sensitive to ciprofloxacin.

In summary, we found that the percentage of decreased antibiotic susceptibility across all species to drugs cefuroxime, cefotaxime and ceftazidime. The most active agents were cefepime and ciprofloxacin. Among them ciprofloxacin was more active than cefepime among all tested organisms except the Acinetobacter spp in which cefepime was more active. So considering the cost and adverse effects of cefepime and ciprofloxacin, the later can be used as first line of drug.

\section{References}

Sievert DM, Ricks P, Edwards JR, et al., "Antimicrobial-resistant pathogens associated with healthcare-associated infections: summary of data reported to the National Healthcare Safety Network at the Centers for Disease Control and Prevention", 2009-2010. Infect Control Hosp Epidemiol. 2013; 34: 1-14.

Bhattacharjee A, Sen M R, Prakash P, Gaur A, Anupurba S. "Increased prevalence of extended spectrum $\beta$ lactamase producers in neonatal septicaemic cases at a tertiary referral hospital". Indian J Med Microbiol [serial online] 2008 [cited 2013 Aug 26]; 26: 356-60.

Shawn R. Lockhart, Murray A. "Abramson, Susan E. Beekmann. Antimicrobial Resistance among Gram-Negative Bacilli Causing Infections in Intensive Care Unit Patients in the United States between 1993 and 2004". J Clin Microbiol. 2007 October; 45(10): 3352-3359.

Bertram G. Katzung, Susan B. Masters, Anthony J. Trevor. "Basic and Clinical Pharmacology" 12edLange; Chapter 43: 797-800.

Bertram G. Katzung, Susan B. Masters, Anthony J. Trevor. "Basic and Clinical Pharmacology" 12edLange; Chapter 46: 834-838.

Ambler RP. "The structure of $\beta$-lactamases". Philos Trans R Soc Lond B 1980; 289: 321-31.

Bush K, Jacoby GA, Medeiros AA. "A functional classification scheme for $\beta$ lactamases and its correlation with molecular structure". Antimicrob Agents Chemother 1995; 39: 1211-33.

Cordron PE, Moland ES, Thomson KS. "Occurrence and detection of AmpC $\beta$ lactamases among E. coli, Klebsiella pneumoniae and Proteus mirabilis isolates at Veterans medical centre". J Clin Microbiol 2000; 38: 1791-6.

Phillipon A, Arlet G, Jacoby GA. "Plasmid determined AmpC type $\beta$-lactamases". Antimicrob Agents Chemother" 2002; 46: 1-11. 
Barnaud G, Arlet G, Verdet C, Gaillot O, Langrange $\mathrm{PH}$, Philippon A. "Salmonella enteritidis AmpC plasmid mediated inducible - $\beta$ - lactamases (DHA-1) with an ampR gene from Morganella morganii". Antimicrob Agents Chemother 1998; 42 : 2352-8.

Thomson KS. "Controversies about extended spectrum and AmpC $\beta$-lactamases". Emerg Infect Dis 2001; 7 : 333-6.

Black JA, Moland ES, Thomson KS. "AmpC disk test for detection of plasmid mediated AmpC $\beta$-lactamases in Enterobacteriaceae lacking chromosomal AmpC $\beta$-lactamases". J Clin Microbiol 2005; 43: 3110-3.

Paterson, D. L., and R. A. Bonomo. "Extended-spectrum beta-lactamases": a clinical update. Clin. Microbiol. Rev 2005; 18:657-686.

Ben-Ami, R., M. J. Schwaber, S. NavonVenezia, D. Schwartz, M. Giladi, I. Chmelnitsky, "Influx of extendedspectrum beta-lactamase-producing Enterobacteriaceae into the hospital". Clin. Infect. Dis 2006; 42:925-934.

Pitout, J. D., P. Nordmann, K. B. Laupland, and L. Poirel. "Emergence of Enterobacteriaceae producing extended-spectrum beta-lactamases (ESBLs) in the community". J. Antimicrob. Chemother 2005; 56:5259.

CLSI. "Performance Standards for Antimicrobial Susceptibility Testing; Twenty Third Information Supplement". CLSI document M100 S23. Wayne, P.A: Clinical and Laboratory Standards Institute; 2013.

Burwen DR, Banerjee SN, Gaynes RP, "The National Nosocomial Infections Surveillance System. Ceftazidime resistance among selected nosocomial gram-negative bacilli in the United States". J Infect Dis.1994; 170: 16221625
Archibald L, Phillips L, Monnet D, McGowan JE, Tenover F, Gaynes R. "Antimicrobial resistance in isolates from inpatients and outpatients in the United States: increasing importance of the intensive care unit". Clin Infect Dis. 1997; 24: 211-215.

Håkan Hanberger, José-Angel GarciaRodriguez, Miguel Gobernado. "Antibiotic Susceptibility Among Aerobic Gram-negative Bacilli in Intensive Care Units in 5 European Countries". JAMA. 1999; 281(1): 6771.

Verbist L. "Incidence of multi-resistance in gram-negative bacterial isolates from intensive care units in Belgium: a surveillance study". Scand J Infect Dis. 1991; 78(suppl): 45-53.

Jarlier V, Fosse T, Philippon A. "Antibiotic susceptibility in aerobic gram-negative bacilli isolated in intensive care units in 39 French teaching hospitals" (ICU study). Intensive Care Med. 1996; 22: 1057-1065.

Livermore DM. "Beta-lactamases in laboratory and clinical resistance". Clin Microbiol Rev. 1995; 8: 557-584.

Philippon A, Labia R, Jacoby G. "Extendedspectrum beta-lactamases". Antimicrob Agents Chemother. 1989; 33:11311136.

Livermore DM, Yuan M. "Antibiotic resistance and production of extendedspectrum beta-lactamases amongst Klebsiella spp from intensive care units in Europe". J Antimicrob Chemother. 1996; 38: 409-424.

Itokazu GS, Quinn JP, Bell-Dixon C. "Antimicrobial resistance rates among gram-negative bacilli recovered from patients in intensive care units: evaluation of a national post marketing surveillance program". Clin Infect Dis. 1996; 23: 779-784.

Jones RN, Marshall SA. "Antimicrobial 
activity of cefepime tested against Bush group I beta-lactamaseproducing strains resistant to ceftazidime. A multilaboratory national and international clinical isolate study". Diagnostic Microbiology and Infectious Disease 1994, 19(1): 33-38.

Christian G. Giske, Dominique L. Monnet, Otto Cars. "Clinical and Economic
Impact of Common MultidrugResistant Gram-Negative Bacilli”. Antimicrob. Agents Chemother. March 2008 vol. 52 no. 3 813-821.

Sinha P, Sharma R, Rishi S. "Prevalence of extended spectrum beta lactamase and AmpC beta lactamase producers among Escherichia coli isolates in a tertiary care hospital in Jaipur". Indian J Pathol Microbiol 2008; 51: 367-9.

\section{How to cite this article:}

Vishwajith, K. Anuradha and Venkatesha, D. 2021. Comparison of Susceptibility of Clinical Isolates of Gram- Negative Bacilli to Cephalosporins and Fluroquinolone. Int.J.Curr.Microbiol.App.Sci. 10(02): 2717-2724. doi: https://doi.org/10.20546/ijcmas.2021.1002.300 